\title{
Head and neck cancer
}

RJ Casasola

Consultant Clinical Oncologist, Tayside Cancer Centre, Dundee, UK

\begin{abstract}
Head and neck cancer is traditionally thought of as being a disease associated with alcohol and tobacco abuse and deprivation. It was the fourth most common cancer in males in Scotland in 2000 and just over I,000 cases were diagnosed in 2007. As smoking becomes less prevalent, a fall in the incidence of head and neck cancer could be anticipated; however, increasing evidence of other aetiological factors contributing to the diagnosis of head and neck cancer, particularly oropharyngeal cancer in non-smoking, little-drinking populations, is impacting on that perception. This review will aim to look at current aetiological factors for head and neck cancer, along with current therapeutic modalities in treatment of this disease.
\end{abstract}

KEYWORDS Head and neck cancer, human papillomavirus

DECLARATION OF INTERESTS No conflict of interests declared.

\section{INTRODUCTION}

Head and neck cancer is currently the fourth most common cancer in males in Scotland. Historically, the typical head and neck cancer patients would be from a group of the population with a high instance of deprivation. They would often give a long history of heavy cigarette smoking and significant alcohol consumption. Treatment for these patients would often involve significantly disfiguring surgery, along with postoperative radiotherapy. Over the past few decades this has changed, with treatments being developed that involve less in the way of mutilating surgery and more induction chemotherapy, concomitant chemo-radiation and more advanced radiation techniques, including conformal therapy and intensity-modulated radiotherapy. In some cases the role of surgery is now being reduced to that of a diagnostic biopsy and perhaps a post-chemoradiation neck dissection. This contrasts with the previous tendency towards radical resection of the primary tumour, along with a radical neck dissection, followed by post-operative radiotherapy.

Increasingly over the past decade or so a second group of head and neck cancer patients has been defined. These are younger than traditional head and neck cancer patients, often in their $40 \mathrm{~s}$ and $50 \mathrm{~s}$, and do not have the same history of cigarette smoking and high alcohol consumption. This group has a different social demographic and a closer male-female incidence. Their tumours are often oropharyngeal, particularly tonsil and base of tongue, are often associated with neck nodal metastases at presentation and have poorly differentiated pathology. It is increasingly recognised that this group of tumours are related to infection with the human papillomavirus (HPV), particularly HPV I6. The evidence certainly suggests that as a group these patients are not only fitter for aggressive treatment from the outset, but do rather better in terms of outcome.

\section{BACKGROUND}

Head and neck cancer is somewhat different from other types of cancer in as much as it, almost inevitably, presents as either a local or a loco-regional disease. It is uncommon for head and neck cancer to present with distant metastases and even head and neck primaries that have metastasised to cervical nodes can still be considered curable. These patients are generally seen in combined clinics and their management considered by both oncologists and surgeons.

Often the aim of treatment from the outset is cure and, in attempting to achieve that, consideration must be given to the various therapeutic modalities to ensure optimal outcomes both in terms of cure, function and cosmesis. For small localised head and neck cancers, optimal treatment will often involve just a single modality; thus a small well-lateralised carcinoma of the tongue could be perfectly well treated by surgical excision, with a high hope of cure and with no significant impact on function or cosmesis. Similarly, a localised carcinoma of the larynx could be treated with radical radiotherapy, again with a high hope of cure and with a good functional and cosmetic outcome.

More advanced disease requires a combination of therapeutic modalities to be used to optimise cure rates and often it will be the sequencing of therapy that differs depending on the site of disease and with the aim of optimising function and cosmesis.

\section{SURGERY VS CHEMO-RADIATION}

The evidence supporting a move away from primary surgery to chemo-radiation came from the US in the 1990s when patients with locally advanced laryngeal cancers were randomised between laryngectomy or induction chemotherapy followed by chemo-radiation to 
those who responded. This showed broadly similar outcomes in terms of survival, with a significant proportion of patients in the induction chemotherapy and chemo-radiation arm retaining a functional larynx.' Patients treated with chemo-radiation who developed a recurrence or had residual disease still had the option of surgical salvage. This led to a similar treatment protocol being applied to patients with disease in other sites of the head and neck, with induction chemotherapy being used as a surrogate for inherent chemo-radiation sensitivity and, for those patients who respond, proceeding with chemo-radiation. This organ-preservation approach has gained fairly wide acceptability but is not without its toxicity. Acute toxicities include xerostomia, mucositis and skin reaction as a consequence of radiotherapy and myelosuppression, nausea and renal impairment as a consequence of chemotherapy. These toxicities often mean that towards the end of a 6.5-week course of chemo-radiation, patients are unable to swallow and often require supplementary feeding either by way of a nasogastric tube or a percutaneous endoscopic gastrostomy (PEG) feeding tube.

Xerostomia after chemo-radiation is very common and patients also report up to $20 \%$ dysphagia rates following chemo-radiation. Because of this significant level of toxicity it is current practice with my own patients who will be undergoing induction chemotherapy and chemoradiation to proceed with PEG tube insertion, peripherally inserted central catheter line insertion and dental assessment before the treatment begins. Of all patients undergoing PEG tube insertion over the past two years, only one has not required supplementary feeding via the tube at some stage during treatment.

For locally advanced disease, the preference in my practice in terms of timing and sequencing of therapeutic modality has been to proceed with induction chemotherapy using a combination of cisplatin and fluorouracil (5FU) for two cycles, followed by radical chemo-radiation, invariably over two phases for advanced disease, with a further two cycles of concomitant cisplatin. Patients who at presentation had $2 \mathrm{~cm}$ or more of measurable cervical adenopathy and those without a complete response receive a post-chemo-radiation neck dissection between 8 and 12 weeks after completing chemo-radiation. Some controversy exists over the sequence of these interventions, some clinicians preferring the surgical approach to the neck in the first instance, followed by definitive chemo-radiation to the primary tumour, and also to the neck as dictated by pathology. There is no evidence to suggest variation in the sequencing of intervention affects outcome, but from a logistical perspective and our own local data supporting the safety of post-chemo-radiation neck dissection, this tends to be the preferred modality locally.

Patients not considered suitable for chemo-radiation can be treated with cetuximab in combination with radiotherapy. This is a monoclonal antibody to the epidermal growth factor receptor that is commonly overexpressed in head and neck cancer. A randomised study has shown that radiotherapy combined with cetuximab is more effective than radiation alone, ${ }^{2}$ but unfortunately there are no studies comparing chemoradiation and cetuximab and radiation.

\section{AETIOLOGICAL FACTORS}

Changes in sexual practice over the past 20-30 years have lead to an increasing number of patients with HPVrelated head and neck cancer. Studies have shown that the risk of HPV-related cancer increases with increasing numbers of sexual and oral sexual partners. ${ }^{3.4}$ Subtyping has shown that HPV 16 is the most common virus implicated, the same as with many cases of cervical cancer. ${ }^{5}$

The increasing recognition of HPV as an aetiological factor in head and neck cancer and the acknowledgement that this patient population has some significant differences from a traditional head and neck cohort has raised some interesting questions. This is a group of patients who are often younger, fitter and with less co-morbidity than typical head and neck cancer patients. There is less association with deprivation and commonly these patients are in gainful employment at the time of their diagnosis. Because of their relatively young age and their need to retain speech, cosmetic and functional outcomes may weigh more heavily in the equation of optimal management.

The evidence certainly suggests that this group of patients has a better prognosis than alcohol- and tobacco-related head and neck cancer, and is better able to withstand the rigours of treatment. Response rates to induction chemotherapy are very high, often with fairly rapid symptomatic response. It will be interesting to see in the coming years how the incidence of HPV-related head and neck cancer changes, given the introduction of HPV vaccination in females. The concern remains, however, that this will not necessarily impact for a decade or longer and, in the interim period, there remains the possibility of a different HPV subtype filling the gap left by immunity to HPV 16 and 18.

\section{REFERENCES}

I Forastiere AA, Goepfert H, Maor M et al. Concurrent chemotherapy and radiotherapy for organ preservation in advanced laryngeal cancer. N Engl J Med 2003; 349:209I-8. doi:I0.1056/NEJMoa03 I 3 I 7
2 Bonner JA, Harari PM, Giralt J et al. Radiotherapy plus cetuximab for squamous-cell carcinoma of the head and neck. $N$ Engl J Med 2006; 354:567-78. doi:I0.1056/NEJMoa053422 
3 Heck JE, Berthiller J, Vaccarella S et al. Sexual behaviours and the risk of head and neck cancers: a pooled analysis in the International Head and Neck Cancer Epidemiology (INHANCE) consortium. Int J Epidemiol 2010; 39:166-8I. doi:I0.1093/ije/dyp350

4 D'Souza G, Kreimer AR, Viscidi R et al. Case-control study of human papillomavirus and oropharyngeal cancer. $N$ Engl J Med 2007; 356:1944-56. doi: I 0.I056/NEJMoa065497
5 Marur S, D'Souza G, Westra WH et al. HPV-associated head and neck cancer: a virus-related cancer epidemic. Lancet Oncol 2010; I I:78I-9. doi:I0.10I6/SI470-2045(I0)700I7-6

\section{SELF-ASSESSMENT QUESTIONS}

I. A 55-year-old man has squamous carcinoma of the base of the tongue with $3 \mathrm{~cm}$ of cervical adenopathy. Which of the following is unlikely to be a relevant aetiological factor?

A. Alcohol.

B. Cigarette smoking.

C. Pipe smoking.

D. Human papillomavirus.

E. Epstein-Barr virus (EBV).

2. A 67-year-old man is diagnosed with TI NO carcinoma of the larynx. Which one of the following is the optimal management?

A. Laryngectomy.

B. Chemo-radiation.

C. Radical radiotherapy.

D. Chemotherapy.

E. Watch and wait.

3. A 46-year-old lawyer presents with a two-month history of right neck swelling. Examination confirms a palpable right level 2 node. Oral cavity appears normal. Which one of the following would be an appropriate action?

A. Review in one month to see if it has settled.

B. A computed tomography scan.

C. Course of antibiotics.

D. Refer to general surgeon for biopsy.

E. Ear, nose, throat (ENT) referral.
4. A 63-year-old heavy smoker presents with a persistent hoarse voice of three months' duration. Which one of the following would be an appropriate action?

A. Review in one month to see if it has settled.

B. Chest X-ray.

C. Course of antibiotics.

D. Refer to respiratory medicine.

E. ENT referral.

5. A 43-year-old accountant presents to the combined clinic with a biopsy-proven, poorly differentiated carcinoma of the oropharynx. Clinically he has a palpable right, level 2 cervical node. With regard to optimal management, which one of the following statements is correct?

A. Radical surgery to the primary site and neck dissection.

B. Radical radiotherapy.

C. Chemo-radiation alone.

D. Chemo-radiation followed by neck dissection.

E. Referral to palliative care.
This paper was originally published as part of the Oncology module in the RCPE Online Continuing Medical Education Programme. Online CME, including the anwers to these questions, is available to Fellows and Members at: http://www.rcpe.ac.uk 